\title{
Paraffinoma induced bilateral preauricular cheek skin defects
}

Jae-Woo Heo ${ }^{1}$,

Baek Kyu Kim ${ }^{2}$

${ }^{1}$ Department of Plastic and

Reconstructive Surgery, Seoul National University Hospital, Seoul National University College of Medicine, Seoul; ${ }^{2}$ Department of Plastic and Reconstructive Surgery, Seoul National University Bundang Hospital, Seoul National University College of Medicine, Seongnam, Korea
"Paraffinoma" is a well-recognized complication of paraffin oil injection into various body parts for an aesthetic purpose. After a variable latency phase, paraffinoma can present as a wide range of clinical symptoms. This paper is a case report of surgical excision of the paraffinoma and subsequent reconstruction of the associated skin defect on bilateral preauricular cheeks, manifesting 50 years after a primary injection.

Keywords: Paraffin oil / Foreign bodies / Granuloma

\section{INTRODUCTION}

First discovered by von Reichenbach in 1830 [1], paraffin is a purified mineral oil whose primary purpose was to serve as a vehicle for oil-soluble substances [2]. Facial augmentation can be achieved using various materials, and in the past, liquid paraffin was deemed a simple and effective method [3]. Liquid paraffin is injected into not only the cheeks, but also other body parts including nose, breasts and penis in order to fill the tissue or contour defects, or purely for an aesthetic volume supplementation [4].

"Paraffinoma" is an adverse consequence of injecting the paraffin oil. Despite being regarded as an unsound therapy, paraffin oil injection is still practiced these days by nonphysicians and cosmetologists not so rarely. In addition, the duration of latent phase is variable, manifesting as long as 10 to 20 years later [5-7]. In this sense, it would not be infrequent to encounter patients with paraffinoma or associated symptoms in a real clini-

Correspondence: Baek Kyu Kim

Department of Plastic and Reconstructive Surgery, Seoul National University Bundang Hospital, Seoul National University College of Medicine, 82 Gumi-ro 173beon-gil, Bundang-gu, Seongnam 13620, Korea

E-mail: plasrecon@gmail.com

Received May 3, 2018 / Revised June 9, 2018 / Accepted June 9, 2018 cal practice nowadays.

In this paper, we are reporting a case of a female patient with a skin defect on bilateral cheeks and exposed foreign body granuloma after paraffin oil injection approximately 50 years ago. No previous case report dealt with bilateral, symmetrical skin defects on preauricular cheeks due to paraffinoma.

\section{CASE REPORT}

An 82-year-old female patient was referred from the Department of Dermatology for a skin defect on bilateral preauricular cheeks. History taking revealed an injection of unidentifiable substances approximately 50 years ago. Over the last 20 years, there has been a progressive skin thinning with telangiectasia on both injection sites. Eventually, since 2006, overlying skin has started to peel off, exposing the underlying foreign body granuloma. Skin defect on both sides gradually increased in their sizes, and at initial presentation, the right and left side defect measured up to $4.0 \times 3.5$ and $3.0 \times 2.5 \mathrm{~cm}$, respectively (Fig. 1). Prior to referral to our clinic, no previous surgical treatment has been attempted.

There was no significant previous medical, other operation or medication history worth of mentioning. Her preoperative 
American Society of Anesthesiologists (ASA) grading was class I. According to the preoperative physical examinations, all facial motors and sensory were normal. Underneath the skin defect on each side, there was a palpable and non-movable masslike lesion.

Preoperative contrast-enhanced computed tomography scan calcifications on bilateral preauricular cheeks, superficial to masseter muscle. Adjacent structures such as parotid gland were deviated from the normal location due to mass effect.

Under general anesthesia, via open defect on each side, the extent and boundary of foreign body were identified. Foreign body was removed, one side at a time, with caution not to dam-

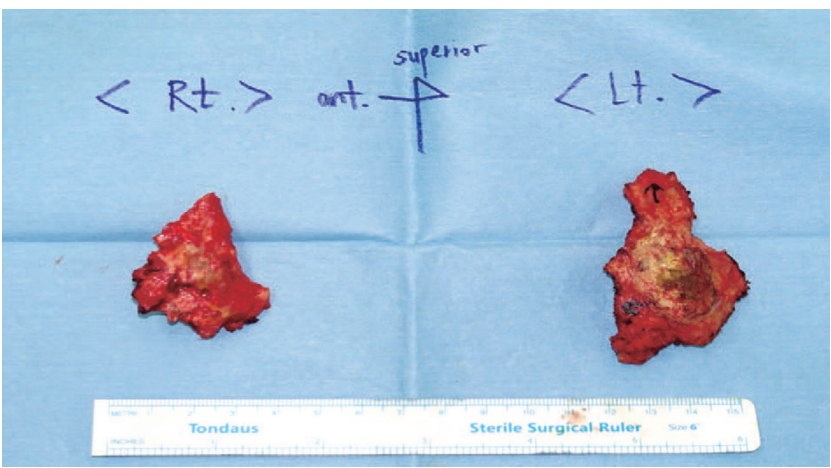

Fig. 2. Surgical specimens. Excised paraffinoma specimen from the right (Rt) and left (Lt) preauricular cheeks.
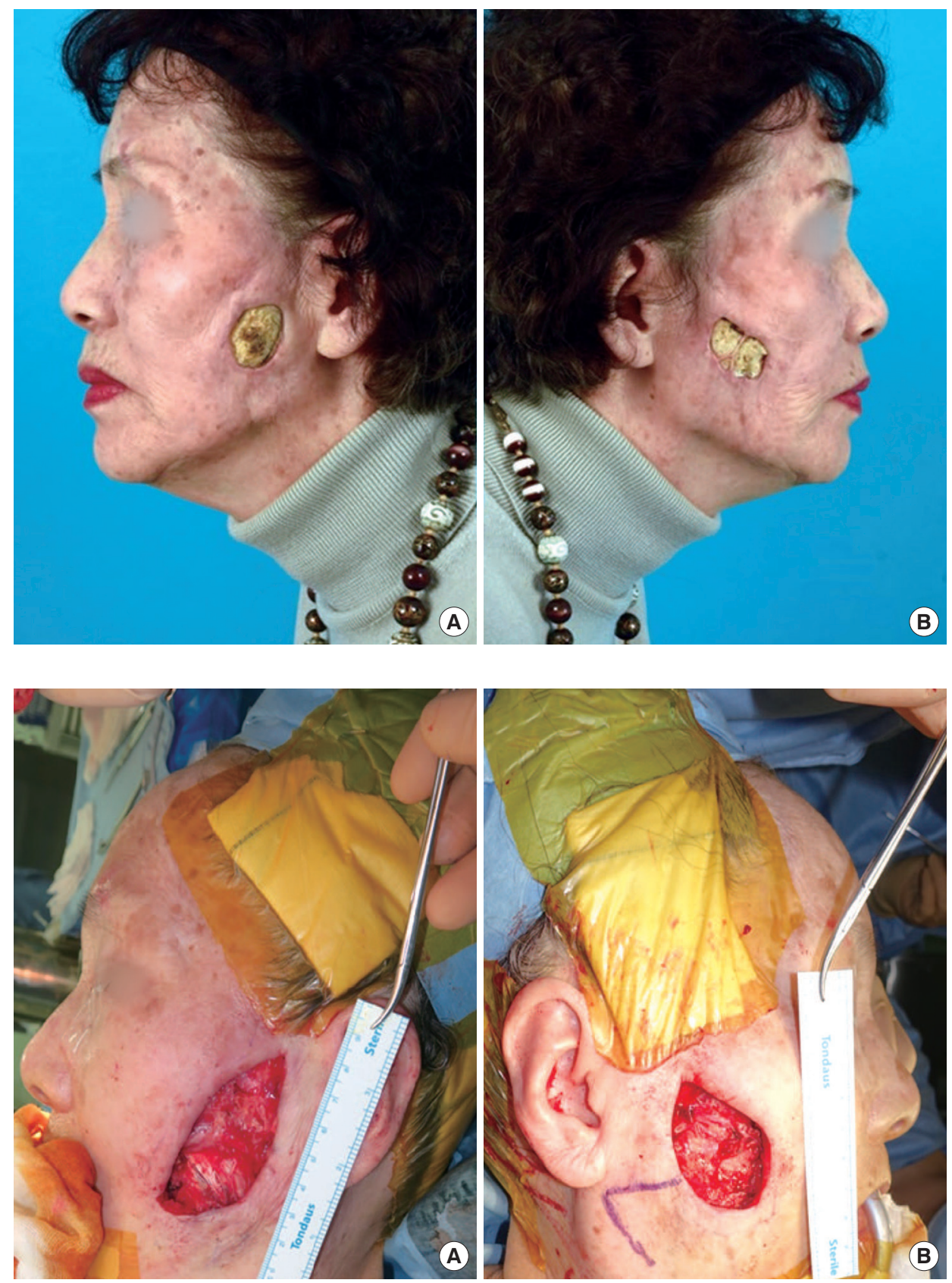

Fig. 1. Preoperative photograph. Bilateral preauricular cheek skin defects with exposed paraffinoma in two different projections. (A) Left and (B) right.

Fig. 3. Remnant skin defect after the removal of paraffinoma. (A) On the left cheek, extended incision was made and primary closure was done. (B) On the right cheek, Limberg flap was used to close the defect. 
age the nearby structures, especially facial nerve main trunk and branches, and parotid gland. During the process of removal, nerve stimulator monitoring guided the navigation of the probable facial nerve location and pathway. Overall, lesions were well-demarcated from the surroundings, and they were completely excised (Fig. 2). Then, each defect margin was debrided. On the left side, an extended incision was made, and primary closure was possible thanks to the skin laxity (Fig. 3A). On the opposite side, however, lower eyelid ectropion was anticipated upon primary closure. Hence, Limberg flap was adopted for the closure of the defect (Fig. 3B). Wound was closed by layers after insertion of a negative-pressure closed-suction

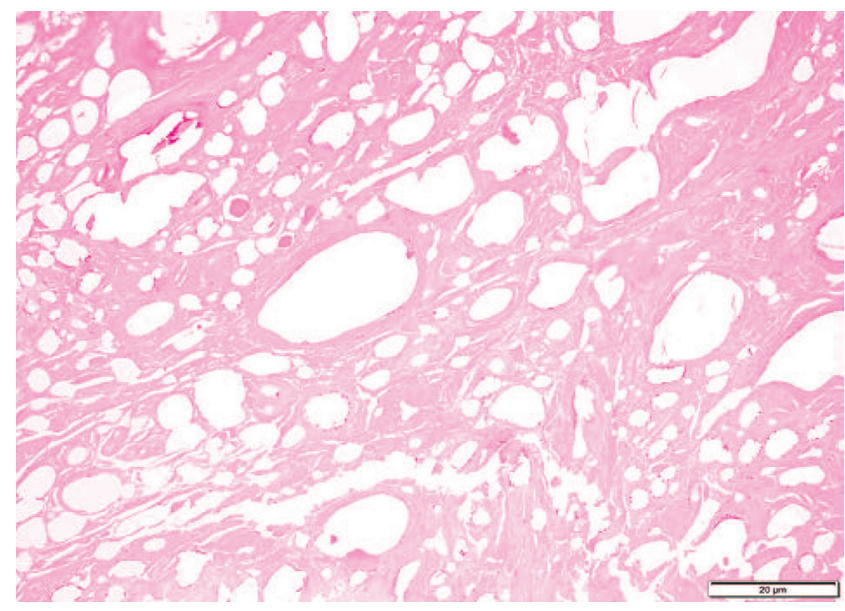

Fig. 4. H\&E stained specimen $(\times 20)$. Round or oval shaped empty pseudo-cysts encompassed by lymphocytes, epithelioid cells and giant cells. drain in each side. Overall, the surgery went uneventful.

During the postoperative periods, skin flaps, especially on the right side was closely monitored. No congestive margins were noted. Postoperative physical examinations revealed no suspicious injury on the facial motor functions. The day after surgery, both drains were removed, and patient was discharged from the hospital. On outpatient clinic, all stitches were removed at the seventh day postoperatively. Permanent biopsy proved both lesions to be consistent with a paraffinoma (Fig. 4). A 4-month follow-up showed a tolerable reconstructive result (Fig. 5).

\section{DISCUSSION}

Paraffinoma is defined as a chronic granulomatous inflammatory reaction [8]. It bears a clinical significance in that it can infiltrate into the nearby structures and initiate all sorts of clinical symptoms such as pain, palpable mass, skin ulceration which can lead to skin defect, sinus opening or fistula formation leading to suppuration, lymphadenopathy, and although very rare, even malignant degeneration [8-12]. Pathological examination of the specimen is the definite diagnostic tool of the paraffinoma. Typical features of paraffinoma in hematoxylin and eosin staining include round or oval shaped empty pseudo-cysts encompassed by lymphocytes, epithelioid cells and giant cells [13]. Paraffinoma is still occasionally encountered in the clinical practice, as some nonphysicians and cosmetologists still practice the injection of paraffin oil these days, and the past injections become clinically apparent in the present days after a sig-

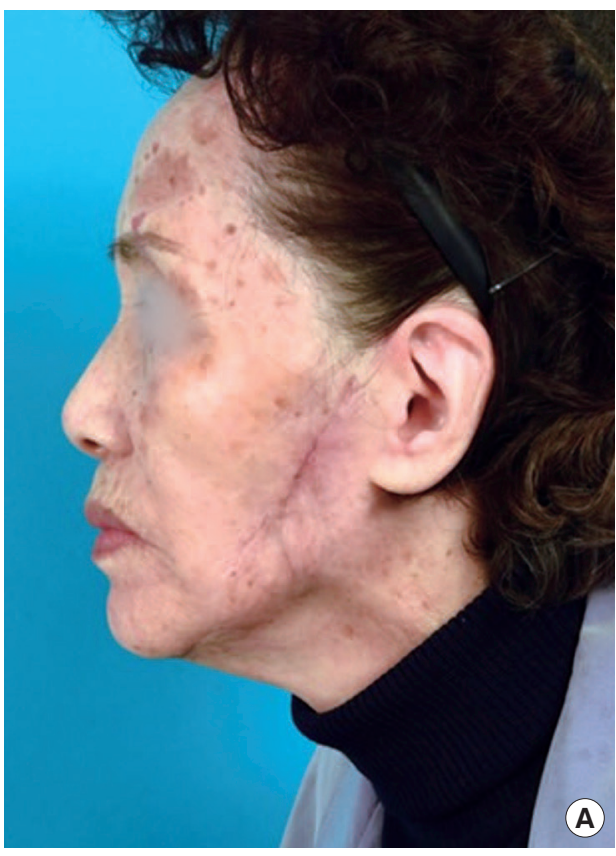

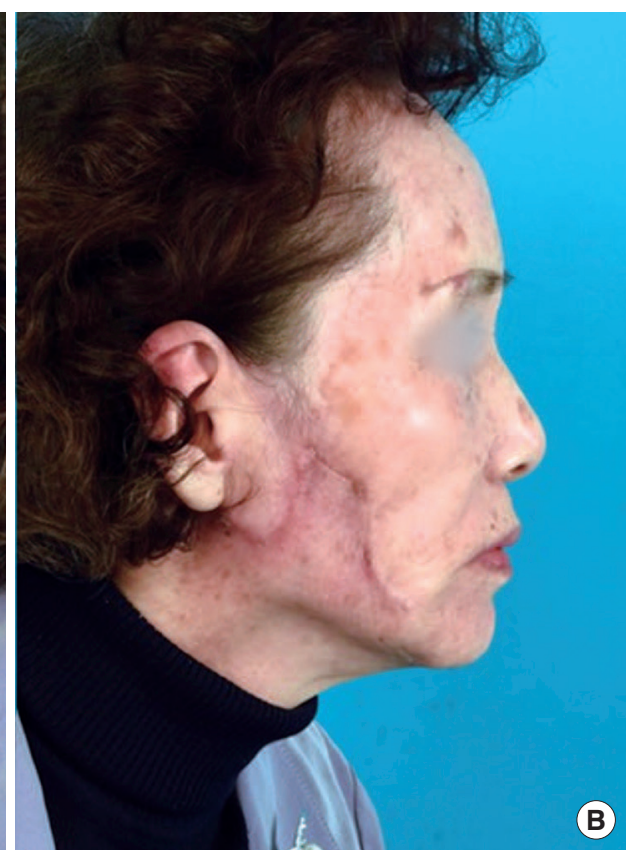

Fig. 5. Postoperative photograph. Four months after the surgery in two different projections. (A) Left and (B) right. 
nificant period of latent phase. The paraffin oil is known to be refractory to all kinds of lysozymal enzymes and substances, and eventually, it forms a chronic inflammatory granuloma, which can cause a wide range of morphological disfigurements including a skin defect, as in this case [8]. Treatment of paraffinoma is still problematic nowadays, especially in the case of a diffuse infiltration into normal adjacent structures [13]. Yet, the golden standard treatment of paraffinoma remains the surgical excision, in most cases $[10,11,14]$. Complete surgical excision is the only way to avoid the recurrence.

In this particular case, the involvement of facial nerve was of the most concern. Moreover, caution was paid not to damage the facial nerve during the process of excision. Preoperative physical examinations revealed no impairment in the facial motors or sensations in the patient. Fortunately, no postoperative impairment was noted. Intraoperatively, structures assumed to be parotid gland and facial nerve main trunk were found to be deviated from their normal location as a result of the paraffinoma's mass effect. However, the margin demarcating the granuloma from these structures was relatively well-defined, and thus, surgical excision came handier than expected.

This paper deals with a clinical case, in which bilateral preauricular cheek skin defects were complicated in an 82-year-old female as a result of a paraffin oil injection 50 years ago. The symmetrical nature of the presentation, very long period of latent phase approximating to 50 years, and finally, successful reconstruction using regional structures whilst preserving the facial motor function all combine to add a clinical significance to the case.

\section{CONFLICT OF INTEREST}

No potential conflict of interest relevant to this article was reported.

\section{PATIENT CONSENT}

The patients provided written informed consent for the publication and the use of their images.

\section{REFERENCES}

1. Goldwyn RM. The paraffin story. Plast Reconstr Surg 1980;65: 517-24.

2. Bloem JJ, Van der Waal I. Paraffinoma of the face. Plast Reconstr Surg 1975;56;473-4.

3. Vazquez-Martinez OT, Ocampo-Candiani J, Mendez-Olvera N, Sanchez Negron FA. Paraffinomas of the facial area: treatment with systemic and intralesional steroids. J Drugs Dermatol 2006;5:186-9.

4. Glicenstein J. The first "fillers", vaseline and paraffin: from miracle to disaster. Ann Chir Plast Esthet 2007;52:157-61.

5. Boo-Chai K. Paraffinoma. Plast Reconstr Surg 1965;36:101-10.

6. Urbach F, Wine SS, Johnson WC, Davies RE. Generalized paraffinoma (sclerosing lipogranuloma). Arch Dermatol 1971;103: 277-85.

7. Winer LH, Sternberg TH, Lehman R, Ashley FL. Tissue reactions to injected silicone liquids: a report of three cases. Arch Dermatol 1964;90:588-93.

8. Legaspi-Vicerra ME, Field LM. Paraffin granulomata, "witch's chin," and nasal deformities: excision and reconstruction with reduction chinplasty and open rhinotomy resection. J Clin Aesthet Dermatol 2010;3:54-8.

9. De Gado F, Mazzocchi M, Chiummariello S, Gagliardi DN, Alfano C. Johnson's baby oil, a new type of filler? Acta Chir Plast 2006;48:123-6.

10. Wong KT, Lee PS, Chan YL, Chow LT. Paraffinoma in anterior abdominal wall mimicking liposarcoma. Br J Radiol 2003;76: 264-7.

11. Erguvan-Dogan B, Yang WT. Direct injection of paraffin into the breast: mammographic, sonographic, and MRI features of early complications. AJR Am J Roentgenol 2006;186:888-94.

12. Colomb D. The future of paraffinomas: apropos of a case of reticulolymphoblasto-sarcomatosis with acquired hemolytic anemia caused by cold auto-antibodies and cryoglobulinemia, having complicated a paraffinoma having 50 years' development. Ann Dermatol Syphiligr (Paris) 1962;89:36-46.

13. Feldmann R, Harms M, Chavaz P, Salomon D, Saurat JH. Orbital and palpebral paraffinoma. J Am Acad Dermatol 1992;26 (5 Pt 2):833-5.

14. Yang WT, Suen M, Ho WS, Metreweli C. Paraffinomas of the breast: mammographic, ultrasonographic and radiographic appearances with clinical and histopathological correlation. Clin Radiol 1996;51:130-3. 